\title{
Reasons for Initiating Canakinumab among Patients with Systemic Juvenile Idiopathic Arthritis and Adult- Onset Still's Disease in the U.S. Real-World Settings
}

\author{
Peter Hur · Esther Yi · Raluca Ionescu-Ittu • Ameur M. Manceur • \\ Kathleen G. Lomax · Jordan Cammarota · Jipan Xie · Raju Gautam • \\ Priscila Nakasato · Navneet Sanghera · Nina Kim • Alexei A. Grom (D)
}

Received: September 23, 2021 / Accepted: November 15, 2021 / Published online: December 7, 2021

(c) The Author(s) 2021

\section{ABSTRACT}

Introduction: The aim of this study was to understand the reasons for canakinumab initiation among patients with Still's disease, including systemic juvenile idiopathic arthritis

Supplementary Information The online version contains supplementary material available at https:// doi.org/10.1007/s40744-021-00402-z.

P. Hur · E. Yi · K. G. Lomax · P. Nakasato

N. Sanghera

Novartis Pharmaceuticals Corporation, East

Hanover, NJ, USA

R. Ionescu-Ittu · A. M. Manceur

Analysis Group, Inc, Montreal, QC, Canada

J. Cammarota

Analysis Group, Inc, Washington, DC, USA

J. Xie

Analysis Group, Inc, Los Angeles, CA, USA

R. Gautam

Novartis Healthcare Pvt. Ltd, Hyderabad, India

N. Kim

Baylor Scott and White Medical Center-Temple,

Temple, TX, USA

N. Kim

The University of Texas at Austin, Austin, TX, USA

A. A. Grom $(\bowtie)$

Division of Pediatric Rheumatology, Children's

Hospital Medical Center, MLC 4010, 3333 Burnet

Avenue, Cincinnati, OH 45229, USA

e-mail: Alexi.Grom@cchmc.org
(SJIA) and adult-onset Still's disease (AOSD), in US clinical practice.

Methods: Physicians retrospectively reviewed the medical charts of patients with Still's disease (regardless of age at symptom onset) who were prescribed canakinumab from 2016 to 2018. Patients aged $<16$ years at symptom onset were classified as having SJIA and those aged $\geq 16$ years at symptom onset (calculated from case-record forms) were classified as having AOSD. Patient treatment history and physician reasons for canakinumab initiation were analyzed. Overall results were presented as SJIA/AOSD. Sensitivity analyses were performed for the robustness of the results.

Results: Forty-three physicians in the USA (rheumatologists/dermatologists/immunologists/allergists: $\quad 51.2 / 27.9 / 11.6 / 9.3 \%$; subspecialty in adults/pediatrics: 67.4/32.6\%) abstracted information for 72 patients with SJIA/AOSD (SJIA/AOSD/age unknown at symptom onset: 75.0/18.1/6.9\%; mean age 19.4 years; children $61.1 \%$; females $56.9 \%$ ). Most patients $(90.3 \%)$ received treatment directly preceding canakinumab initiation (etanercept 27.7\%; anakinra 18.5\%; adalimumab $16.9 \%$ ); the respective treatment was discontinued due to lack of efficacy/effectiveness $(43.1 \%)$ and availability of a new treatment $(27.8 \%)$. Most common reasons for canakinumab initiation were physician perceived/experienced efficacy/effectiveness of canakinumab (77.8\%; children/adults: 81.8/71.4\%), lack-of- 
response to previous treatment $(45.8 \%$; children/adults: $36.4 / 60.7 \%$ ), convenient administration/dosing (26.4\%; children/adults: 29.5/ $21.4 \%$ ) and ability to discontinue/spare steroids (25.0\%; children/adults: 20.5/32.1\%). The sensitivity analysis provided similar results.

Conclusions: In US clinical practice, physician perceived/experienced efficacy/effectiveness of canakinumab and lack-of-response to previous treatment were the primary reasons for canakinumab initiation among patients with SJIA/ AOSD. Physician perceived/experienced efficacy/effectiveness and convenient administration/dosing of canakinumab were the most common reasons for canakinumab initiation among children, whereas lack-of-response to previous treatment and ability to discontinue/ spare steroids being the most frequent reasons among adults.

Keywords: Adult-onset Still's disease; Canakinumab; Medical charts; Real-world; Systemic juvenile idiopathic arthritis

\section{Key Summary Points}

What is already known about this subject?

Canakinumab, a human monoclonal antibody, has demonstrated efficacy and safety in clinical trials in patients with systemic juvenile idiopathic arthritis (SJIA) and adult-onset Still's disease (AOSD).

Canakinumab has been approved in the USA and Europe for the treatment of active Still's disease (including AOSD and SJIA in patients aged $\geq 2$ years). However, there is limited research on prescribing patterns among physicians who initiate canakinumab in real-world settings.

\section{What does this study add?}

Review of medical charts of 72 patients with SJIA/AOSD in this real-world study from the USA revealed that physician perceived/experienced

efficacy/effectiveness of canakinumab and patient's lack-of-response to previous treatment were the most common reasons for canakinumab initiation in US clinical practice.

The study also revealed that physician perceived/experienced efficacy/effectiveness and convenient administration/dosing of canakinumab were more frequent reasons for canakinumab initiation among children, whereas lack-of-response to previous treatment and ability to discontinue/spare steroids were more common reasons among adults.

\section{How might this impact on clinical practice or future developments?}

The study highlighted differences in the reasons for canakinumab initiation among children and adults, as well as by subspecialty of the treating physician, but further research is needed to better understand the explanations behind this prescription behavior.

Since this study was not designed to follow-up the patient after canakinumab initiation, the exact reasons for change in initial/maintenance dosing from the approved label could not be ascertained and further research is suggested in this area.

\section{INTRODUCTION}

Systemic juvenile idiopathic arthritis (SJIA), also referred to as Still's disease, is a rare auto-inflammatory disease characterized by arthritis and significant extra-articular systemic features, 
including fever, evanescent rash, lymphadenopathy, hepatosplenomegaly and polyserositis [1]. SJIA is the most severe subset of juvenile idiopathic arthritis (JIA), accounting for approximately $10.0 \%$ of JIA cases in North America and Europe $[2,3]$. The peak age of SJIA onset is 1-5 years, although it can occur at any age during childhood. As part of the same Still's disease spectrum, adult-onset Still's disease (AOSD) is the nomenclature given if onset is at or after 16 years of age, and its incidence is very low (i.e. 0.16 cases per 100,000 person) [4].

SJIA differs from other well-known childhood arthritic conditions (which are classic autoimmune diseases) by being an auto-inflammatory condition, with the pro-inflammatory cytokines interleukin (IL)- $1 \beta$ and IL-6 identified as key mediators in its pathogenesis $[5,6]$. SJIA is associated with a substantial disease burden, with children experiencing disability, structural joint damage and reduced health-related quality of life [7]. Both SJIA and AOSD impose a high physical, psychological and financial burden not only on patients but also on their families [3].

Treatment goals in Still's disease are attaining symptomatic relief, controlling the inflammation and achieving and maintaining sustained disease remission [2]. Both conventional and biological disease-modifying antirheumatic drugs (DMARDs) are used in SJIA and AOSD. Patients are often initially treated with non-steroidal anti-inflammatory drugs (NSAIDs) followed by oral corticosteroids (OCS), if symptoms persist. Of note, the DMARD methotrexate is not effective for the treatment of systemic features of SJIA or AOSD, but might be an effective treatment for the arthritic features $[2,8]$. Therapies targeting IL- 1 and IL-6 cytokines have demonstrated significant efficacy towards managing systemic manifestations of Still's disease [2]. In 2011, tocilizumab, an IL6 inhibitor, became the first biological therapy to be approved in the USA for SJIA [9].

Canakinumab, a human monoclonal antibody effective against IL-1 $\beta$, has been approved in the USA and Europe for the treatment of active Still's disease, including AOSD and SJIA in patients aged $\geq 2$ years $[10,11]$. Clinical trials have demonstrated the efficacy and safety of canakinumab in patients with SJIA and AOSD [12-14]. However, there is limited research on the disease and treatment characteristics of canakinumab-treated patients and prescribing patterns among physicians who initiate canakinumab in real-world settings. The initial aim of our study was to to characterize the clinical and treatment profiles of only patients with SJIA regardless of their age at symptom onset (with no intention/option to include patients with AOSD) who were prescribed canakinumab and the physician reasons for canakinumab initiation in US clinical practice. The analysis was expanded to include AOSD after data collection showed the presence of a cohort of these patients.

\section{METHODS}

\section{Study Design}

This was a retrospective chart review involving patients with Still's disease treated with canakinumab by different specialist physicians (allergists, dermatologists, immunologists and rheumatologists) in the USA. An external vendor invited physicians from their panel of medical specialists representing all US states to participate; all invitations were sent by e-mail. Participating physicians included those who had prescribed canakinumab to $\geq 1$ patient with Still's disease from 2016 to 2018 and agreed to extract patient data in an online case report form (CRF). The methods are described in detail elsewhere [15].

\section{Patient Selection}

Patients were considered eligible if they started canakinumab therapy for the treatment of Still's disease (regardless of age at symptom onset) in the past 24 months from the chart review date and had medical records (including disease characteristics and treatment history) accessible to the participating physicians from the date of first canakinumab prescription. Patients who had initiated canakinumab in a clinical trial were excluded. Included patients were classified 
as patients with SJIA if their age was $<16$ years at the time of symptom onset; those aged $\geq 16$ years at time of symptom onset were classified as having AOSD in accordance with published criteria [16]. Thus, both patients with SJIA and AOSD were included, and the overall findings for all patients were presented as SJIA/ AOSD.

\section{Data Collection}

Participating physicians retrospectively collected data from the medical records of eligible patients in an online CRF. Information on patient demographics, disease diagnosis, clinical characteristics, treatment history and reasons for discontinuing the previous treatments and initiating canakinumab were extracted. Additionally, details on physician specialty, type and region of practice and years in practice were collected. An external vendor was involved in entering extracted data into a common database that was subsequently transferred to the authors for analysis, thereby assuring patient confidentiality. Overall, data collection process was completed in a double blind manner such that the identities of physicians and sponsor were not disclosed to each other. The New England Independent Review Board (NEIRB \#120180302) approved the study prior to the initiation of data collection. The study was conducted in accordance with the Helsinki Declaration of 1964 and its later amendments.

\section{Statistical Analysis}

Patient characteristics were summarized using descriptive statistics. Continuous variables were described as mean (standard deviation) or median (interquartile range) and categorical variables as number (percentage). To minimize occurrence of missing values, physicians were not allowed to leave a question unanswered and where applicable, response options "not applicable", "unknown" and/or "free-text information" were included. Therefore, missing values were not imputed. In addition, quality control measures were taken involving consistency checks, range checks and electronic and manual verification to ensure accuracy of data collected in the CRFs. Subgroup analyses included results stratified according to patient age at the time of canakinumab initiation (children $[<18$ years old] and adults) and subspecialty of the treating physician (pediatric and adult).

\section{Sensitivity Analysis}

The robustness of the results was checked by a sensitivity analysis performed on a subset of the study sample after excluding the patients with AOSD as well as any adult patients with unknown age at symptom onset. An additional sensitivity analysis was performed for the subset of patients with AOSD.

\section{RESULTS}

In total, 43 physicians participated, which is an overall response rate of $<15.0 \%$. These physicians contributed 72 medical charts of patients with SJIA/AOSD, with on average 1.7 charts/physician. Based on the age at symptom onset, there were $54(75.0 \%)$ patients with SJIA and $13(18.1 \%)$ with AOSD (with an additional 5 [6.9\%] patients whose age at symptom onset was unknown). Based on the age at canakinumab initiation, the patients were stratified to children (44 patients) and adults (28 patients) (Table 1).

\section{Physician Characteristics}

The mean age of physicians who filled the CRF was 45.6 years, and their medical specialty was rheumatology $(51.2 \%)$, dermatology $(27.9 \%)$, immunology $(11.6 \%)$ or allergy $(9.3 \%)$ with primary subspecialty in adults $(67.4 \%)$ or pediatrics (32.6\%; Electronic Supplementary Material [ESM] Table S1). Rheumatology was the most common medical specialty of the treating physicians who prescribed canakinumab for both children and adults (each 57.0\%). Other than rheumatologists, adults were often treated by immunologists ( 25.0 vs. $2.3 \%)$, whereas children were mostly treated by dermatologists (17.9 vs. $36.4 \%$ ). Notably, $61.4 \%$ of children 
Table 1 Disease diagnosis information

\begin{tabular}{|c|c|c|c|c|c|}
\hline \multirow[t]{2}{*}{ Disease diagnosis factors } & \multirow{2}{*}{$\begin{array}{l}\text { Overall study } \\
\text { population } \\
(N=72)\end{array}$} & \multirow[t]{2}{*}{$\begin{array}{l}\text { Children } \\
(N=44)\end{array}$} & \multirow[t]{2}{*}{$\begin{array}{l}\text { Adults } \\
(N=28)\end{array}$} & \multicolumn{2}{|c|}{$\begin{array}{l}\text { By treating physician } \\
\text { subspecialty }\end{array}$} \\
\hline & & & & $\begin{array}{l}\text { Pediatric } \\
\text { subspecialty } \\
(N=25)\end{array}$ & $\begin{array}{l}\text { Adult } \\
\text { subspecialty } \\
(N=47)\end{array}$ \\
\hline $\begin{array}{l}\text { Age at SJIA/AOSD diagnosis (years), } \\
\text { mean (SD) }\end{array}$ & $14.0(12.6)$ & $8.0(3.8)$ & $23.5(15.5)$ & $9.4(6.0)$ & $16.5(14.4)$ \\
\hline \multicolumn{6}{|l|}{ SJIA/AOSD type, $n(\%)$} \\
\hline $\begin{array}{l}\text { Active arthritis and active systemic } \\
\text { features }\end{array}$ & $51(70.8)$ & $34(77.3)$ & $17(60.7)$ & $20(80.0)$ & $31(66.0)$ \\
\hline $\begin{array}{l}\text { Active arthritis and no active systemic } \\
\text { features }\end{array}$ & $21(29.2)$ & $10(22.7)$ & $11(39.3)$ & $5(20.0)$ & $16(34.0)$ \\
\hline \multicolumn{6}{|c|}{ Time elapsed between initial symptoms and diagnosis, $n(\%)$} \\
\hline$<6$ months & $17(23.6)$ & $11(25.0)$ & $6(21.4)$ & $12(48.0)$ & $5(10.6)$ \\
\hline $6-12$ months & $20(27.8)$ & $14(31.8)$ & $6(21.4)$ & $3(12.0)$ & $17(36.2)$ \\
\hline $1-2$ years & $18(25.0)$ & $6(13.6)$ & $12(42.9)$ & $6(24.0)$ & $12(25.5)$ \\
\hline $2-5$ years & $8(11.1)$ & $7(15.9)$ & $1(3.6)$ & $2(8.0)$ & $6(12.8)$ \\
\hline$>5$ years & $8(11.1)$ & $6(13.6)$ & $2(7.1)$ & $2(8.0)$ & $6(12.8)$ \\
\hline Unknown & $1(1.4)$ & $0(0.0)$ & $1(3.6)$ & $0(0.0)$ & $1(2.1)$ \\
\hline \multicolumn{6}{|c|}{ Specialty of physician who has first diagnosed SJIA/AOSD, $n(\%)$} \\
\hline Rheumatology & $40(55.6)$ & $27(61.4)$ & $13(46.4)$ & $17(68.0)$ & $23(48.9)$ \\
\hline Immunology & $10(13.9)$ & $3(6.8)$ & $7(25.0)$ & $4(16.0)$ & $6(12.8)$ \\
\hline Internal medicine & $9(12.5)$ & $7(15.9)$ & $2(7.1)$ & $0(0.0)$ & $9(19.1)$ \\
\hline Dermatology & $7(9.7)$ & $5(11.4)$ & $2(7.1)$ & $3(12.0)$ & $4(8.5)$ \\
\hline Allergy & $2(2.8)$ & $1(2.3)$ & $1(3.6)$ & $1(4.0)$ & $1(2.1)$ \\
\hline Cardiology & $1(1.4)$ & $0(0.0)$ & $1(3.6)$ & $0(0.0)$ & $1(2.1)$ \\
\hline Unknown & $3(4.2)$ & $1(2.3)$ & $2(7.1)$ & $0(0.0)$ & $3(6.4)$ \\
\hline \multicolumn{6}{|l|}{ Methods of diagnosis, $n(\%)$} \\
\hline $\begin{array}{l}\text { Assessment of clinical manifestations } \\
\text { and complications (e.g. recurrent } \\
\text { fever) }\end{array}$ & $66(91.7)$ & $43(97.7)$ & $23(82.1)$ & $25(100.0)$ & $41(87.2)$ \\
\hline $\begin{array}{l}\text { Exclusion/rule-out diagnostics (e.g. } \\
\text { infection, neoplasms) }\end{array}$ & $54(75.0)$ & $39(88.6)$ & $15(53.6)$ & $19(76.0)$ & $35(74.5)$ \\
\hline Age of onset & $48(66.7)$ & $32(72.7)$ & $16(57.1)$ & $11(44.0)$ & $37(78.7)$ \\
\hline $\begin{array}{l}\text { Laboratory assessments (e.g. CRP, } \\
\text { ESR, SAA) }\end{array}$ & $37(51.4)$ & $25(56.8)$ & $12(42.9)$ & $14(56.0)$ & $23(48.9)$ \\
\hline
\end{tabular}


Table 1 continued

\begin{tabular}{|c|c|c|c|c|c|}
\hline \multirow[t]{2}{*}{ Disease diagnosis factors } & \multirow{2}{*}{$\begin{array}{l}\text { Overall study } \\
\text { population } \\
(N=72)\end{array}$} & \multirow[t]{2}{*}{$\begin{array}{l}\text { Children } \\
(N=44)\end{array}$} & \multirow[t]{2}{*}{$\begin{array}{l}\text { Adults } \\
(N=28)\end{array}$} & \multicolumn{2}{|c|}{$\begin{array}{l}\text { By treating physician } \\
\text { subspecialty }\end{array}$} \\
\hline & & & & $\begin{array}{l}\text { Pediatric } \\
\text { subspecialty } \\
(N=25)\end{array}$ & $\begin{array}{l}\text { Adult } \\
\text { subspecialty } \\
(N=47)\end{array}$ \\
\hline $\begin{array}{l}\text { Assessment of triggers (e.g. } \\
\text { menstruation, vaccination, stress, } \\
\text { cold, infection) }\end{array}$ & $25(34.7)$ & $14(31.8)$ & $11(39.3)$ & $7(28.0)$ & $18(38.3)$ \\
\hline Assessment of family history/ancestry & $23(31.9)$ & $14(31.8)$ & $9(32.1)$ & $8(32.0)$ & $15(31.9)$ \\
\hline Response to trial therapy & $18(25.0)$ & $8(18.2)$ & $10(35.7)$ & $4(16.0)$ & $14(29.8)$ \\
\hline Genetic tests & $4(5.6)$ & $0(0.0)$ & $4(14.3)$ & $2(8.0)$ & $2(4.3)$ \\
\hline Other $^{a}$ & $1(1.4)$ & $0(0.0)$ & $1(3.6)$ & $1(4.0)$ & $2(4.3)$ \\
\hline \multicolumn{6}{|c|}{ Diagnoses ruled out prior to the confirmed diagnosis, $n$ (\%) } \\
\hline Fever of unknown origin & $55(76.4)$ & $37(84.1)$ & $18(64.3)$ & $19(76.0)$ & $36(76.6)$ \\
\hline Other juvenile idiopathic arthritis & $31(43.1)$ & $14(31.8)$ & $17(60.7)$ & $10(40.0)$ & $21(44.7)$ \\
\hline Other periodic fever syndrome & $28(38.9)$ & $19(43.2)$ & $9(32.1)$ & $10(40.0)$ & $18(38.3)$ \\
\hline $\begin{array}{l}\text { Vasculitis (e.g. polyarthritis nodosa, } \\
\text { Behcet's disease) }\end{array}$ & $24(33.3)$ & $14(31.8)$ & $10(35.7)$ & $8(32.0)$ & $16(34.0)$ \\
\hline Rheumatoid arthritis & $22(30.6)$ & $16(36.4)$ & $6(21.4)$ & $5(20.0)$ & $17(36.2)$ \\
\hline Systemic lupus erythematosus & $15(20.8)$ & $8(18.2)$ & $7(25.0)$ & $5(20.0)$ & $10(21.3)$ \\
\hline Pharyngitis & $15(20.8)$ & $11(25.0)$ & $4(14.3)$ & $5(20.0)$ & $10(21.3)$ \\
\hline Neoplasms & $15(20.8)$ & $7(15.9)$ & $8(28.6)$ & $4(16.0)$ & $11(23.4)$ \\
\hline Urticaria or rash/allergy & $14(19.4)$ & $6(13.6)$ & $8(28.6)$ & $4(16.0)$ & $10(21.3)$ \\
\hline Other & $17(23.6)$ & $12(27.3)$ & $5(17.9)$ & $7(28.0)$ & $10(21.3)$ \\
\hline No rule-out diagnosis & $2(2.8)$ & $2(4.5)$ & $0(0.0)$ & $1(4.0)$ & $1(2.1)$ \\
\hline
\end{tabular}

AOSD Adult-onset Still's Disease, CRP C-reactive protein, $E S R$ erythrocyte sedimentation rate, $N$ total number of patients in the respective category, $S A A$ serum amyloid A, $S D$ standard deviation, SJIA systemic juvenile idiopathic arthritis

ather methods of diagnosis included "X rays"

were prescribed canakinumab by adult subspecialists (Table 2). Majority of physician were in private practice $(74.4 \%)$ and $39.5 \%$ were from Western USA. On average, each physician had 15.6 years of experience; cared for an average of 21.7 patients with SJIA/AOSD in the past 24 months; and prescribed canakinumab to 5.8 patients on average (ESM Table S1).

\section{SJIA/AOSD Diagnosis and Patient Characteristics at Canakinumab Initiation}

At the time of SJIA/AOSD diagnosis, $70.8 \%$ of patients had both active arthritis and active systemic features (children 77.3\%; adults $60.7 \%$ ). The time elapsed between initial presentation of symptoms and SJIA/AOSD diagnosis was up to 1 year in $51.4 \%$ of patients (children 56.8\%; adults $42.8 \%$ ) and $1-5$ years in 
Table 2 Patient characteristics, disease characteristics and clinical manifestations at canakinumab initiation

\begin{tabular}{|c|c|c|c|c|c|}
\hline \multirow[t]{2}{*}{ Characteristics } & \multirow{2}{*}{$\begin{array}{l}\text { Overall study } \\
\text { population } \\
(N=72)\end{array}$} & \multirow[t]{2}{*}{$\begin{array}{l}\text { Children } \\
(N=44)\end{array}$} & \multirow[t]{2}{*}{$\begin{array}{l}\text { Adults } \\
(N=28)\end{array}$} & \multicolumn{2}{|c|}{$\begin{array}{l}\text { By treating physician } \\
\text { subspecialty }\end{array}$} \\
\hline & & & & $\begin{array}{l}\text { Pediatric } \\
\text { subspecialty } \\
(N=25)\end{array}$ & $\begin{array}{l}\text { Adult } \\
\text { subspecialty } \\
(N=47)\end{array}$ \\
\hline Age (years), mean (SD) & $19.4(15.4)$ & $10.4(3.9)$ & $33.5(16.1)$ & $15.7(11.9)$ & $21.3(16.7)$ \\
\hline$<18$ years, $n(\%)$ & $44(61.1)$ & $44(100.0)$ & $0(0.0)$ & $17(68.0)$ & $27(57.4)$ \\
\hline Female, $n(\%)$ & $41(56.9)$ & $25(56.8)$ & $16(57.1)$ & $13(52.0)$ & $28(59.6)$ \\
\hline \multirow[t]{2}{*}{ Height $(\mathrm{m})$, mean $(\mathrm{SD})$} & $1.4(0.3)$ & $1.3(0.3)$ & $1.7(0.2)$ & $1.3(0.4)$ & $1.3(0.3)$ \\
\hline & {$[N=60]$} & {$[N=39]$} & {$[N=21]$} & {$[N=25]$} & {$[N=45]$} \\
\hline \multirow[t]{2}{*}{ Weight $(\mathrm{kg})$, mean $(\mathrm{SD})$} & $51.1(25.3)$ & $36.7(17.8)$ & $74.2(17.0)$ & $44.6(23.2)$ & $53.4(25.6)$ \\
\hline & {$[N=60]$} & {$[N=39]$} & {$[N=21]$} & {$[N=25]$} & {$[N=45]$} \\
\hline \multirow[t]{2}{*}{ BMI (for adults only; $\mathrm{kg} / \mathrm{m}^{2}$ ), mean (SD) } & $26.5(6.1)$ & - & $26.5(6.1)$ & $31.7(15.2)$ & $40.9(25.9)$ \\
\hline & {$[N=20]$} & & {$[N=20]$} & {$[N=8]$} & {$[N=18]$} \\
\hline \multicolumn{6}{|l|}{ Race/ethnicity, $n$ (\%) } \\
\hline White/non-Hispanic & $49(68.1)$ & $26(59.1)$ & $23(82.1)$ & $17(68.0)$ & $32(68.1)$ \\
\hline Hispanic & $11(15.3)$ & $8(18.2)$ & $3(10.7)$ & $3(12.0)$ & $8(17.0)$ \\
\hline Asian/Pacific Islander & $8(11.1)$ & $8(18.2)$ & $0(0.0)$ & $2(8.0)$ & $6(12.8)$ \\
\hline Black/non-Hispanic & $4(5.6)$ & $2(4.5)$ & $2(7.1)$ & $3(12.0)$ & $1(2.1)$ \\
\hline \multicolumn{6}{|l|}{ Insurance type, $n(\%)$} \\
\hline Commercial/private & $59(81.9)$ & $34(77.3)$ & $25(89.3)$ & $21(84.0)$ & $38(80.9)$ \\
\hline Medicare & $10(13.9)$ & $0(0.0)$ & $2(7.1)$ & $0(0.0)$ & $2(4.3)$ \\
\hline Medicaid & $2(2.8)$ & $8(18.2)$ & $2(7.1)$ & $4(16.0)$ & $6(12.8)$ \\
\hline Military & $2(2.8)$ & $2(4.5)$ & $0(0.0)$ & $0(0.0)$ & $2(4.3)$ \\
\hline \multicolumn{6}{|c|}{ Primary medical specialty of the physician who prescribed canakinumab, $n(\%)$} \\
\hline Rheumatology & $41(56.9)$ & $25(56.8)$ & $16(57.1)$ & $9(36.0)$ & $32(68.1)$ \\
\hline Dermatology & $21(29.2)$ & $16(36.4)$ & $5(17.9)$ & $7(28.0)$ & $14(29.8)$ \\
\hline Allergy & $2(2.8)$ & $2(4.5)$ & $0(0.0)$ & $2(8.0)$ & $0(0.0)$ \\
\hline Immunology & $8(11.1)$ & $1(2.3)$ & $7(25.0)$ & $7(28.0)$ & $1(2.1)$ \\
\hline \multicolumn{6}{|c|}{ Primary subspecialty of the physician who prescribed canakinumab, $n$ (\%) } \\
\hline Adult subspecialty & $47(65.3)$ & $27(61.4)$ & $20(71.4)$ & $0(0.0)$ & $47(100.0)$ \\
\hline Pediatric subspecialty & $25(34.7)$ & $17(38.6)$ & $8(28.6)$ & $25(100.0)$ & $0(0.0)$ \\
\hline Years from SJIA diagnosis to canakinumab initiation & $0.7(0.8)$ & $0.7(0.8)$ & $0.8(0.6)$ & $0.8(0.8)$ & $0.7(0.8)$ \\
\hline $\begin{array}{l}\text { reported among patients diagnosed with SJIA/AOSD in } \\
\text { year } 2016 \text { or later, mean (SD) }\end{array}$ & {$[N=42]$} & {$[N=32]$} & {$[N=10]$} & {$[N=16]$} & {$[N=26]$} \\
\hline \multicolumn{6}{|l|}{ SJIA severity, $n$ (\%) } \\
\hline Mild & $8(11.1)$ & $4(9.1)$ & $4(14.3)$ & $3(12.0)$ & $5(10.6)$ \\
\hline
\end{tabular}


Table 2 continued

\begin{tabular}{|c|c|c|c|c|c|}
\hline \multirow[t]{2}{*}{ Characteristics } & \multirow{2}{*}{$\begin{array}{l}\text { Overall study } \\
\text { population } \\
(N=72)\end{array}$} & \multirow[t]{2}{*}{$\begin{array}{l}\text { Children } \\
(N=44)\end{array}$} & \multirow[t]{2}{*}{$\begin{array}{l}\text { Adults } \\
(N=28)\end{array}$} & \multicolumn{2}{|c|}{$\begin{array}{l}\text { By treating physician } \\
\text { subspecialty }\end{array}$} \\
\hline & & & & $\begin{array}{l}\text { Pediatric } \\
\text { subspecialty } \\
(N=25)\end{array}$ & $\begin{array}{l}\text { Adult } \\
\text { subspecialty } \\
(N=47)\end{array}$ \\
\hline Moderate & $54(75.0)$ & $34(77.3)$ & $20(71.4)$ & $19(76.0)$ & $35(74.5)$ \\
\hline Severe & $10(13.9)$ & $6(13.6)$ & $4(14.3)$ & $3(12.0)$ & $7(14.9)$ \\
\hline \multicolumn{6}{|l|}{ Number of joints with active inflammation, $n$ (\%) } \\
\hline None & $4(5.6)$ & $4(9.1)$ & $0(0.0)$ & $2(8.0)$ & $2(4.3)$ \\
\hline Mono/oligoarthritis (1-4 joints) & $50(69.4)$ & $30(68.2)$ & $20(71.4)$ & $18(72.0)$ & $32(68.1)$ \\
\hline Polyarthritis ( $\geq 5$ joints) & $18(25.0)$ & $10(22.7)$ & $8(28.6)$ & $5(20.0)$ & $13(27.7)$ \\
\hline \multicolumn{6}{|l|}{ Number of joints with limited range of motion, $n$ (\%) } \\
\hline None & $15(20.8)$ & $13(29.5)$ & $2(7.1)$ & $2(8.0)$ & $13(27.7)$ \\
\hline $1-4$ joints & $45(62.5)$ & $24(54.5)$ & $21(75.0)$ & $18(72.0)$ & $27(57.4)$ \\
\hline$\geq 5$ joints & $11(15.3)$ & $6(13.6)$ & $5(17.9)$ & $4(16.0)$ & $7(14.9)$ \\
\hline Unknown & $1(1.4)$ & $1(2.3)$ & $0(0.0)$ & $1(4.0)$ & $0(0.0)$ \\
\hline \multicolumn{6}{|c|}{ SJIA/AOSD clinical manifestations at canakinumab initiation, $n(\%)$} \\
\hline Fever & $52(72.2)$ & $36(81.8)$ & $16(57.1)$ & $18(72.0)$ & $34(72.3)$ \\
\hline Fatigue/malaise & $42(58.3)$ & $24(54.5)$ & $18(64.3)$ & $13(52.0)$ & $29(61.7)$ \\
\hline $\begin{array}{l}\text { Skin/cutaneous (e.g. rash, other skin/cutaneous } \\
\text { manifestations) }\end{array}$ & $34(47.2)$ & $25(56.8)$ & $9(32.1)$ & $7(28.0)$ & $27(57.4)$ \\
\hline $\begin{array}{l}\text { Musculoskeletal (e.g. arthritis, other musculoskeletal } \\
\text { manifestations) }\end{array}$ & $33(45.8)$ & $17(38.6)$ & $16(57.1)$ & $8(32.0)$ & $25(53.2)$ \\
\hline Liver abnormalities & $8(11.1)$ & $6(13.6)$ & $2(7.1)$ & $0(0.0)$ & $8(17.0)$ \\
\hline $\begin{array}{l}\text { Mood/behavior (e.g. depression/anxiety, appetite/taste } \\
\text { alterations) }\end{array}$ & $8(11.1)$ & $5(11.4)$ & $3(10.7)$ & $2(8.0)$ & $6(12.8)$ \\
\hline $\begin{array}{l}\text { Lymphoid organ (e.g. lymphadenopathy, hepatomegaly, } \\
\text { splenomegaly) }\end{array}$ & $7(9.7)$ & $4(9.1)$ & $3(10.7)$ & $2(8.0)$ & $5(10.6)$ \\
\hline Pharyngitis & $5(6.9)$ & $4(9.1)$ & $1(3.6)$ & $1(4.0)$ & $4(8.5)$ \\
\hline Cardiorespiratory/circulatory organ & $2(2.8)$ & $1(2.3)$ & $1(3.6)$ & $1(4.0)$ & $1(2.1)$ \\
\hline Complications of SJIA/AOSD & $1(1.4)$ & $0(0.0)$ & $1(3.6)$ & $1(4.0)$ & $0(0.0)$ \\
\hline Other $^{\mathrm{a}}$ & $2(2.8)$ & $2(4.5)$ & $0(0.0)$ & $1(4.0)$ & $1(2.1)$ \\
\hline None of the above & $6(8.3)$ & $2(4.5)$ & $4(14.3)$ & $1(4.0)$ & $5(10.6)$ \\
\hline
\end{tabular}

$B M I$ Body mass index

a Other types of clinical manifestations included "elevated inflammatory markers" and "rise in ferritin" 
36.1\% (children 29.5\%; adults 46.5\%). The three main methods used for diagnosis included clinical manifestations and complications (91.7\%), exclusion/rule-out diagnostics (75.0\%) and age of onset $(66.7 \%$; Table 1$)$.

At the time of canakinumab initiation, the mean age of overall patients was 19.4 years (61.1\% children; $56.9 \%$ females); the mean age was 10.4 years for children and 33.5 years for adults. On average, the duration of disease from diagnosis to canakinumab initiation was 5.4 years (children 2.4 years; adults 10.0 years). Fever (72.2\%), fatigue/malaise (58.3\%), skin/cutaneous (47.2\%) and musculoskeletal manifestations $(45.8 \%)$ were the most common signs/symptoms. While fever (81.8 vs. $57.1 \%)$ and skin/cutaneous signs/symptoms (56.8 vs. $32.1 \%$ ) were more common in children versus adults, fatigue/malaise (54.5 vs. $64.3 \%)$ and musculoskeletal manifestations (38.6 vs. $57.1 \%$ ) were more prevalent in adults. Patients had one to four joints with active inflammation (69.4\%) and one to four joints with limited range of motion $(62.5 \%)$, with both features being more common among adults than children (Table 2).

Table 3 Patients with SJIA/AOSD treated with a long-term treatment directly preceding canakinumab initiation

\begin{tabular}{|c|c|c|c|c|c|}
\hline \multirow{2}{*}{$\begin{array}{l}\text { Treatments directly preceding } \\
\text { canakinumab initiation, } n(\%)\end{array}$} & \multirow{2}{*}{$\begin{array}{l}\text { Overall study } \\
\text { population } \\
(N=65)\end{array}$} & \multirow{2}{*}{$\begin{array}{l}\text { Children } \\
(N=38)\end{array}$} & \multirow{2}{*}{$\begin{array}{l}\text { Adults } \\
(N=27)\end{array}$} & \multicolumn{2}{|c|}{ By treating physician subspecialty } \\
\hline & & & & $\begin{array}{l}\text { Pediatric } \\
\text { subspecialty } \\
(N=25)\end{array}$ & $\begin{array}{l}\text { Adult } \\
\text { subspecialty } \\
(N=40)\end{array}$ \\
\hline Methotrexate & $6(9.2)$ & $4(10.5)$ & $2(7.4)$ & $3(12.0)$ & $3(7.5)$ \\
\hline $\begin{array}{l}\text { Non-steroidal anti-inflammatory } \\
\text { drugs }\end{array}$ & $5(7.7)$ & $3(7.9)$ & $2(7.4)$ & $1(4.0)$ & $4(10.0)$ \\
\hline Oral corticosteroids & $4(6.2)$ & $3(7.9)$ & $1(3.7)$ & $0(0.0)$ & $4(10.0)$ \\
\hline Corticosteroid injection & $2(3.1)$ & $2(5.3)$ & $0(0.0)$ & $2(8.0)$ & $0(0.0)$ \\
\hline Leflunomide & $1(1.5)$ & $0(0.0)$ & $1(3.7)$ & $0(0.0)$ & $1(2.5)$ \\
\hline $\begin{array}{l}\text { Calcineurin inhibitors (e.g. } \\
\text { cyclosporine) }\end{array}$ & $0(0.0)$ & $0(0.0)$ & $0(0.0)$ & $0(0.0)$ & $0(0.0)$ \\
\hline Thalidomide & $0(0.0)$ & $0(0.0)$ & $0(0.0)$ & $0(0.0)$ & $0(0.0)$ \\
\hline \multicolumn{6}{|l|}{ Biologics } \\
\hline Etanercept & $18(27.7)$ & $8(21.1)$ & $10(37.0)$ & $4(16.0)$ & $14(35.0)$ \\
\hline Anakinra & $12(18.5)$ & $5(13.2)$ & $7(25.9)$ & $5(20.0)$ & $7(17.5)$ \\
\hline Adalimumab & $11(16.9)$ & $7(18.4)$ & $4(14.8)$ & $2(8.0)$ & $9(22.5)$ \\
\hline Tocilizumab & $7(10.8)$ & $4(10.5)$ & $3(11.1)$ & $4(16.0)$ & $3(7.5)$ \\
\hline Abatacept & $3(4.6)$ & $1(2.6)$ & $2(7.4)$ & $3(12.0)$ & $0(0.0)$ \\
\hline Infliximab & $2(3.1)$ & $2(5.3)$ & $0(0.0)$ & $0(0.0)$ & $2(5.0)$ \\
\hline Rilonacept & $1(1.5)$ & $0(0.0)$ & $1(3.7)$ & $0(0.0)$ & $1(2.5)$ \\
\hline Rituximab & $0(0.0)$ & $0(0.0)$ & $0(0.0)$ & $0(0.0)$ & $0(0.0)$ \\
\hline Unknown & $5(7.7)$ & $5(13.2)$ & $0(0.0)$ & $5(20.0)$ & $0(0.0)$ \\
\hline
\end{tabular}

Patients who did not receive this line of therapy and patients who received canakinumab as first treatment are excluded Treatment agents used are not mutually exclusive 
a

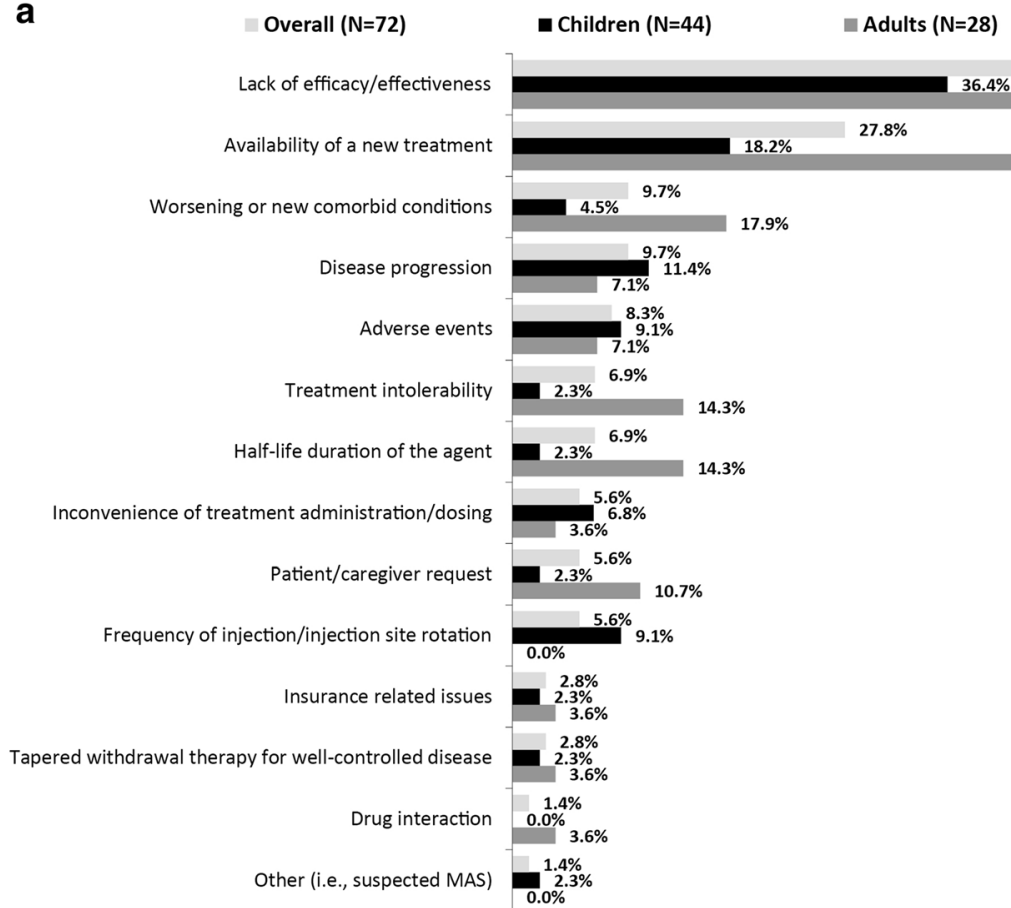

b $\quad$ Treated at pediatric subspecialty ( $\mathrm{N}=25) \quad$ Treated at adult subspeciality ( $\mathrm{N}=47)$

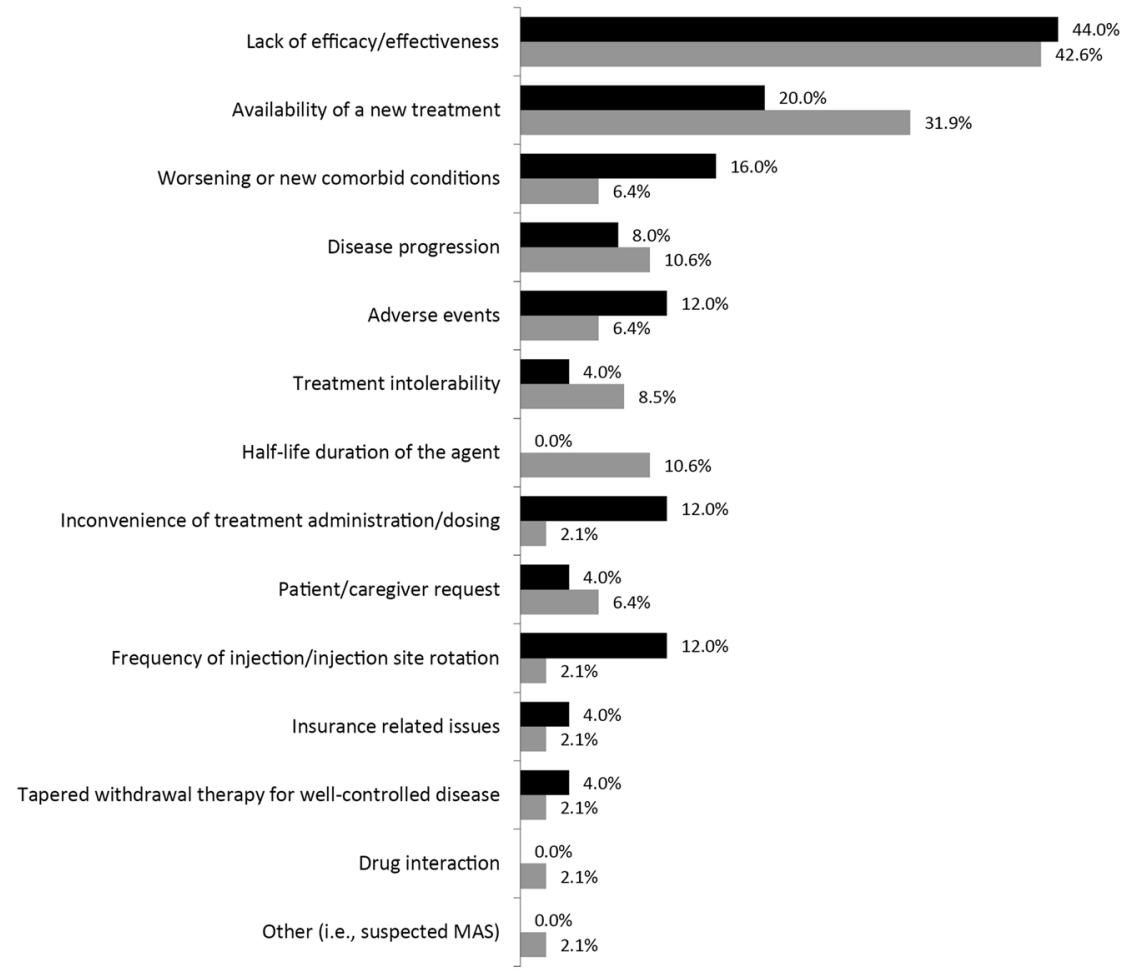


4Fig. 1 Reasons for discontinuation of treatment prior to canakinumab: a Children and adults, b By treating physician subspecialty. Note: More than one reason per patient possible. MAS Macrophage activation syndrome, $N$ total number of patients in the respective category

\section{Treatment History Prior to Canakinumab}

Nearly all physicians (97.7\%) followed treatment guidelines while treating patients, among whom $62.8 \%$ referred to "Up-To-Date" guidelines, $60.5 \%$ to American College of Rheumatology guidelines and $53.5 \%$ to peer-reviewed articles. Information on first long-term treatment for SJIA/AOSD was available for 67 (93.1\%) patients, with information on NSAIDs (47.2\%), OCS $(43.1 \%)$, methotrexate $(43.1 \%)$ and anakinra (20.8\%) being the most common. In the subgroup by physician type, patients treated by pediatric subspecialists more commonly received anakinra (28.0 vs. $17.0 \%)$, whereas those treated by adult subspecialists more frequently received NSAIDs (36.0 vs. $53.2 \%)$, OCS ( 28.0 vs. $51.1 \%$ ) or methotrexate (32.0 vs. $48.9 \%$; ESM Tables S1, S2).

Biologics, including etanercept $(27.7 \%)$, anakinra (18.5\%), adalimumab (16.9\%) and tocilizumab $(10.8 \%)$, were the common treatments directly preceding canakinumab initiation. The use of etanercept (37.0 vs. $21.1 \%$ ) and anakinra (25.9 vs. $13.2 \%)$ was more frequent among adults versus children. In the subgroup treated by pediatric subspecialists, more patients received anakinra (20.0 vs. $17.5 \%)$ and tocilizumab (16.0 vs. $7.5 \%)$, while those treated by adult subspecialists more commonly received etanercept (16.0 vs. $35.0 \%)$ and adalimumab ( 8.0 vs. $22.5 \%$; Table 3 ).

\section{Reasons for Discontinuation of Treatments Prior to Canakinumab}

Among the 72 patients with Still's disease, the most shared reasons for treatment discontinuation prior to canakinumab were the lack of efficacy/effectiveness (43.1\%) and availability of a new treatment $(27.8 \%)$. Among children versus adults, disease progression (11.4 vs. $7.1 \%)$ and high frequency of injection/need for frequent rotation of the injection site (9.1 vs. $0.0 \%$ ) were also the notable reasons for treatment discontinuation prior to canakinumab; whereas worsening/new comorbid conditions ( 4.5 vs. $17.9 \%)$, treatment intolerability ( 2.3 vs. $14.3 \%)$ and short half-life duration of the agent (2.3 vs. $14.3 \%)$ were other common reasons among adults (Fig. 1a). In the subgroup treated by pediatric subspecialists, patients more commonly discontinued treatment due to worsening/new comorbid conditions, adverse events (AEs), inconvenience of treatment administration/dosing and high frequency of injection/ need for frequent rotation of the injection site, while patients treated by adult subspecialists discontinued the treatment more often due to the availability of a new treatment and short half-life duration of the agent (Fig. 1b).

Of note, per specific biologic used preceding canakinumab, the lack of efficacy/effectiveness was the reason for discontinuation among all patients $(100.0 \%)$ treated with adalimumab $(N=11)$ and abatacept $(N=3), 57.1 \%$ of those treated with tocilizumab $(N=7)$ and $50.0 \%$ of those treated with etanercept $(N=18)$. The availability of a new treatment was the reason for discontinuation in $66.7 \%$ of patients treated with etanercept and $41.7 \%$ of those treated with anakinra $(N=12)$. Short half-life duration of the agent and high frequency of injection/need for frequent rotation of the injection site was the reason for discontinuation of treatment among $25.0 \%$ of patients each treated with anakinra. Etanercept was discontinued in $22.2 \%$ of patients due to AEs (Table 4).

\section{Canakinumab Prescribing Pattern}

The median initial dose of canakinumab was $150.0 \mathrm{mg}$, irrespective of the age of patient at treatment initiation or specialty of the treating physician. On average, patients received $144.3 \mathrm{mg}$ (children $132.0 \mathrm{mg}$; adults $151.6 \mathrm{mg}$ ) or $2.9 \mathrm{mg} / \mathrm{kg}$ (children $3.3 \mathrm{mg} / \mathrm{kg}$; adults $2.3 \mathrm{mg} / \mathrm{kg}$ ) of canakinumab as the initial dose. The average canakinumab initial dose was $2.7 \mathrm{mg} / \mathrm{kg}$ for patients treated by adult subspecialists and $3.3 \mathrm{mg} / \mathrm{kg}$ for those treated by 
Table 4 Reasons for discontinuation of the treatments prior to canakinumab-for overall patients by biological agents

\begin{tabular}{|c|c|c|c|c|c|c|}
\hline $\begin{array}{l}\text { Specific reasons listed (not } \\
\text { mutually exclusive), } n \text { (\%) }\end{array}$ & $\begin{array}{l}\text { Etanercept } \\
(N=18)\end{array}$ & $\begin{array}{l}\text { Anakinra } \\
(N=12)\end{array}$ & $\begin{array}{l}\text { Adalimumab } \\
(N=11)\end{array}$ & $\begin{array}{l}\text { Tocilizumab } \\
(N=7)\end{array}$ & $\begin{array}{l}\text { Abatacept } \\
(N=3)\end{array}$ & $\begin{array}{l}\text { Infliximab } \\
(N=2)\end{array}$ \\
\hline Lack of efficacy/effectiveness & $9(50.0)$ & $2(16.7)$ & $11(100.0)$ & $4(57.1)$ & $3(100.0)$ & $0(0.0)$ \\
\hline Availability of a new treatment & $12(66.7)$ & $5(41.7)$ & $0(0.0)$ & $1(14.3)$ & $0(0.0)$ & $1(50.0)$ \\
\hline $\begin{array}{l}\text { Inconvenience of treatment } \\
\text { administration/dosing }\end{array}$ & $0(0.0)$ & $2(16.7)$ & $0(0.0)$ & $1(14.3)$ & $0(0.0)$ & $0(0.0)$ \\
\hline Disease progression & $3(16.7)$ & $0(0.0)$ & $2(18.2)$ & $1(14.3)$ & $0(0.0)$ & $1(50.0)$ \\
\hline Half-life duration of the agent & $1(5.6)$ & $3(25.0)$ & $0(0.0)$ & $0(0.0)$ & $0(0.0)$ & $0(0.0)$ \\
\hline Treatment intolerability & $2(11.1)$ & $2(16.7)$ & $0(0.0)$ & $0(0.0)$ & $0(0.0)$ & $0(0.0)$ \\
\hline $\begin{array}{l}\text { Worsening or new comorbid } \\
\text { conditions }\end{array}$ & $2(11.1)$ & $2(16.7)$ & $1(9.1)$ & $0(0.0)$ & $0(0.0)$ & $1(50.0)$ \\
\hline Adverse events & $4(22.2)$ & $0(0.0)$ & $0(0.0)$ & $0(0.0)$ & $0(0.0)$ & $0(0.0)$ \\
\hline $\begin{array}{l}\text { Frequency of injection/injection } \\
\text { site rotation }\end{array}$ & $0(0.0)$ & $3(25.0)$ & $0(0.0)$ & $0(0.0)$ & $0(0.0)$ & $0(0.0)$ \\
\hline Patient/caregiver request & $3(16.7)$ & $1(8.3)$ & $0(0.0)$ & $0(0.0)$ & $0(0.0)$ & $1(50.0)$ \\
\hline Insurance-related issues & $0(0.0)$ & $1(8.3)$ & $0(0.0)$ & $1(14.3)$ & $0(0.0)$ & $0(0.0)$ \\
\hline $\begin{array}{l}\text { Tapered withdrawal therapy for } \\
\text { well-controlled disease }\end{array}$ & $0(0.0)$ & $0(0.0)$ & $0(0.0)$ & $2(28.6)$ & $0(0.0)$ & $0(0.0)$ \\
\hline Other cost-related reasons & $0(0.0)$ & $0(0.0)$ & $0(0.0)$ & $0(0.0)$ & $0(0.0)$ & $0(0.0)$ \\
\hline Drug interaction & $1(5.6)$ & $0(0.0)$ & $0(0.0)$ & $0(0.0)$ & $0(0.0)$ & $0(0.0)$ \\
\hline $\begin{array}{l}\text { Patient/caregiver's social } \\
\text { environment (i.e., rural/urban) }\end{array}$ & $0(0.0)$ & $0(0.0)$ & $0(0.0)$ & $0(0.0)$ & $0(0.0)$ & $0(0.0)$ \\
\hline Other & $1(5.6)$ & $0(0.0)$ & $0(0.0)$ & $0(0.0)$ & $0(0.0)$ & $0(0.0)$ \\
\hline
\end{tabular}

For rilonacept and rituximab, either the treatment was not discontinued or it was never taken before initiating canakinumab

pediatric subspecialists. Regarding the frequency of dosing, $51.4 \%$ used it every 4 weeks (q4w; children $45.5 \%$; adults $60.7 \%$ ) and $48.6 \%$ used it every 8 weeks (q8w; children $54.5 \%$; adults $39.3 \%$ ). In patients treated by pediatric subspecialists canakinumab $\mathrm{q} 8 \mathrm{w}$ was more common (56.0 vs. $44.0 \%)$, whereas in those treated by adult subspecialists $\mathrm{q} 4 \mathrm{w}$ dosing was more frequent (55.3 vs. $44.7 \%$; Table 5).

\section{Reasons for Canakinumab Initiation}

The decision of canakinumab initiation was made by both physician and caregiver/patient $(72.2 \%)$, by physician only $(26.4 \%)$ or by the caregiver/patient only (1.4\%). Overall, the physician perceived/experienced efficacy/effectiveness of canakinumab (77.8\%) and lack-ofresponse to previous treatment $(45.8 \%)$ were the most common reasons for canakinumab initiation. Physician perceived/experienced efficacy/effectiveness (81.8 vs. $71.4 \%)$ and convenient administration/dosing (29.5 vs. $21.4 \%$ ) 
Table 5 Canakinumab initiation patterns

\begin{tabular}{|c|c|c|c|c|c|}
\hline \multirow[t]{2}{*}{$\begin{array}{l}\text { Canakinumab use } \\
\text { characteristics }\end{array}$} & \multirow[t]{2}{*}{$\begin{array}{l}\text { All patients } \\
(N=72)\end{array}$} & \multirow[t]{2}{*}{$\begin{array}{l}\text { Children } \\
(N=44)\end{array}$} & \multirow[t]{2}{*}{$\begin{array}{l}\text { Adults } \\
(N=28)\end{array}$} & \multicolumn{2}{|c|}{$\begin{array}{l}\text { By treating physician } \\
\text { subspecialty }\end{array}$} \\
\hline & & & & $\begin{array}{l}\text { Pediatric } \\
\text { subspecialty } \\
(N=25)\end{array}$ & $\begin{array}{l}\text { Adult } \\
\text { subspecialty } \\
(N=47)\end{array}$ \\
\hline $\begin{array}{l}\text { Age when first prescribed } \\
\text { canakinumab (years), mean } \\
\text { (SD) }\end{array}$ & $18.3(15.3)$ & $9.4(3.7)$ & $32.4(16.0)$ & $15.0(11.7)$ & $20.1(16.7)$ \\
\hline \multicolumn{6}{|c|}{ Year of canakinumab initiation, $n$ (\%) } \\
\hline 2016 & $2(2.8)$ & $1(2.3)$ & $1(3.6)$ & $0(0.0)$ & $2(4.3)$ \\
\hline 2017 & $27(37.5)$ & $15(34.1)$ & $12(42.9)$ & $10(40.0)$ & $17(36.2)$ \\
\hline 2018 & $43(59.7)$ & $28(63.6)$ & $15(53.6)$ & $15(60.0)$ & $28(59.6)$ \\
\hline $\begin{array}{l}\text { Initial dose (reported by } \\
\text { physician as } \mathrm{mg} / \mathrm{kg} \text { or } \\
\text { calculated } \mathrm{mg} / \mathrm{kg} \text { when } \\
\text { weight was available), mean } \\
\text { (SD) }\end{array}$ & $\begin{array}{l}2.9(1.8) \\
{[N=71]}\end{array}$ & {$[N=44]$} & $\begin{array}{l}2.3(1.4) \\
{[N=27]}\end{array}$ & $\begin{array}{l}3.3(2.1) \\
{[N=24]}\end{array}$ & {$[N=47]$} \\
\hline $\begin{array}{l}\text { Initial dose among physicians } \\
\text { who reported } \mathrm{mg} / \mathrm{kg} \text {, mean } \\
\text { (SD) }\end{array}$ & $\begin{array}{l}3.1(1.7) \\
{[N=37]}\end{array}$ & $\begin{array}{l}3.1(1.8) \\
{[N=31]}\end{array}$ & $\begin{array}{l}3.0(1.1) \\
{[N=6]}\end{array}$ & $\begin{array}{l}3.5(2.5) \\
{[N=14]}\end{array}$ & $\begin{array}{l}2.8(1.0) \\
{[N=23]}\end{array}$ \\
\hline $\begin{array}{l}\text { Initial dose among physicians } \\
\text { who reported } \mathrm{mg}\end{array}$ & $N=35$ & $N=13$ & $N=22$ & $N=11$ & $N=24$ \\
\hline Mean (SD) & $144.3(73.6)$ & $132.0(68.7)$ & $151.6(77.0)$ & $153.6(82.4)$ & $140.1(70.8)$ \\
\hline Median (Q1, Q3) & $\begin{array}{l}150.0 \\
\quad(150.0-150.0)\end{array}$ & $\begin{array}{l}150.0 \\
\quad(80.0-150.0)\end{array}$ & $\begin{array}{l}150.0 \\
\quad(150.0-150.0)\end{array}$ & $\begin{array}{l}150.0 \\
\quad(80.0-150.0)\end{array}$ & $\begin{array}{l}150.0 \\
\quad(150.0-150.0)\end{array}$ \\
\hline \multicolumn{6}{|l|}{ Initial frequency, $n$ (\%) } \\
\hline Every 4 weeks & $37(51.4)$ & $20(45.5)$ & $17(60.7)$ & $11(44.0)$ & $26(55.3)$ \\
\hline Every 8 weeks & $35(48.6)$ & $24(54.5)$ & $11(39.3)$ & $14(56.0)$ & $21(44.7)$ \\
\hline \multicolumn{6}{|c|}{ Received concomitant treatment with another long or short-term SJIA/AOSD medication (including biologics), $n$ (\%) } \\
\hline Yes & $19(26.4)$ & $11(25.0)$ & $8(28.6)$ & $3(12.0)$ & $16(34.0)$ \\
\hline No & $53(73.6)$ & $33(75.0)$ & $20(71.4)$ & $22(88.0)$ & $31(66.0)$ \\
\hline
\end{tabular}

Q1 First quartile, Q3 third quartile

of canakinumab were more often the reasons for canakinumab initiation among children versus adults, whereas lack-of-response to previous treatment (36.4 vs. $60.7 \%)$ and ability to discontinue/spare steroids (20.5 vs. $32.1 \%$ ) were more frequent reasons among adults (Fig. 2a). In the subgroup treated by adult subspecialists, lack-of-response to previous treatment (59.6 vs. $20.0 \%$ ), ability to discontinue/spare steroids (31.9 vs. $12.0 \%)$ and half-life duration of 
a

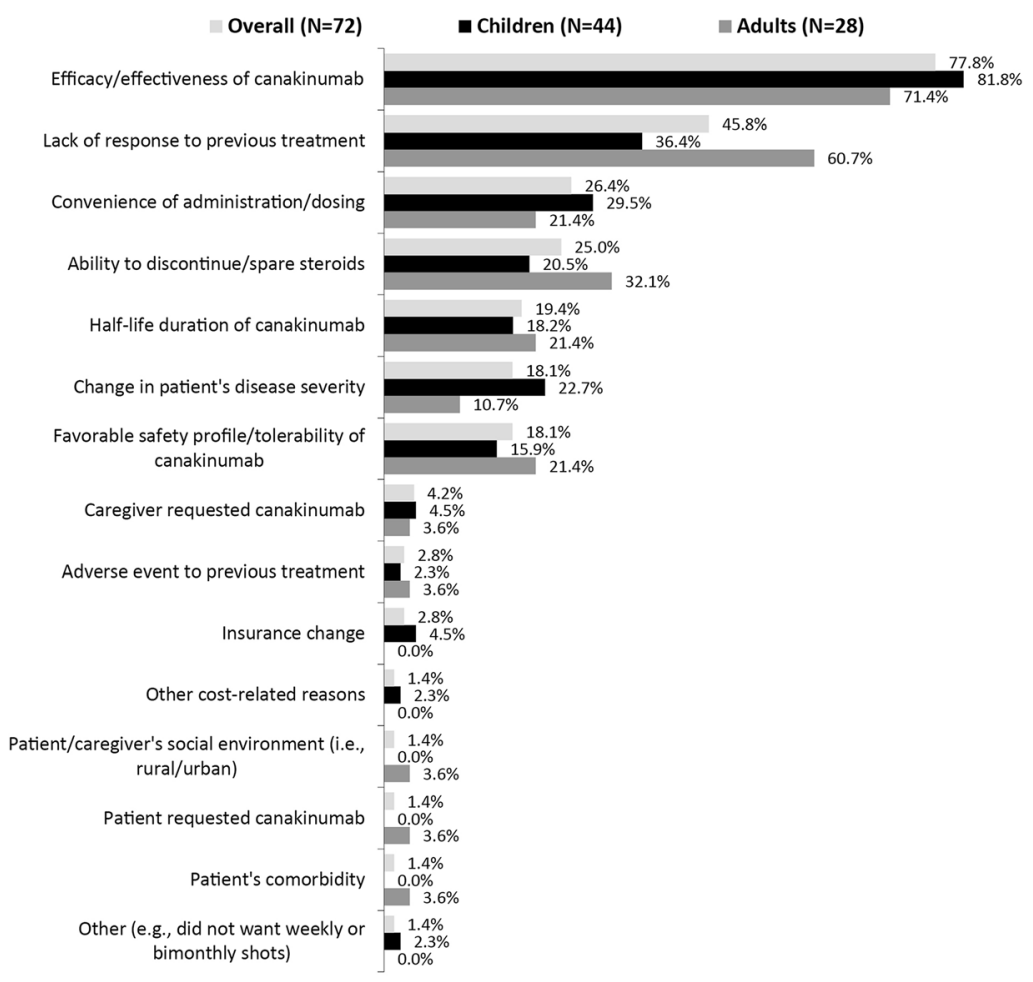

b

- Treated at pediatric subspecialty $(\mathrm{N}=25) \quad \square$ Treated at adult subspeciality $(\mathrm{N}=47)$

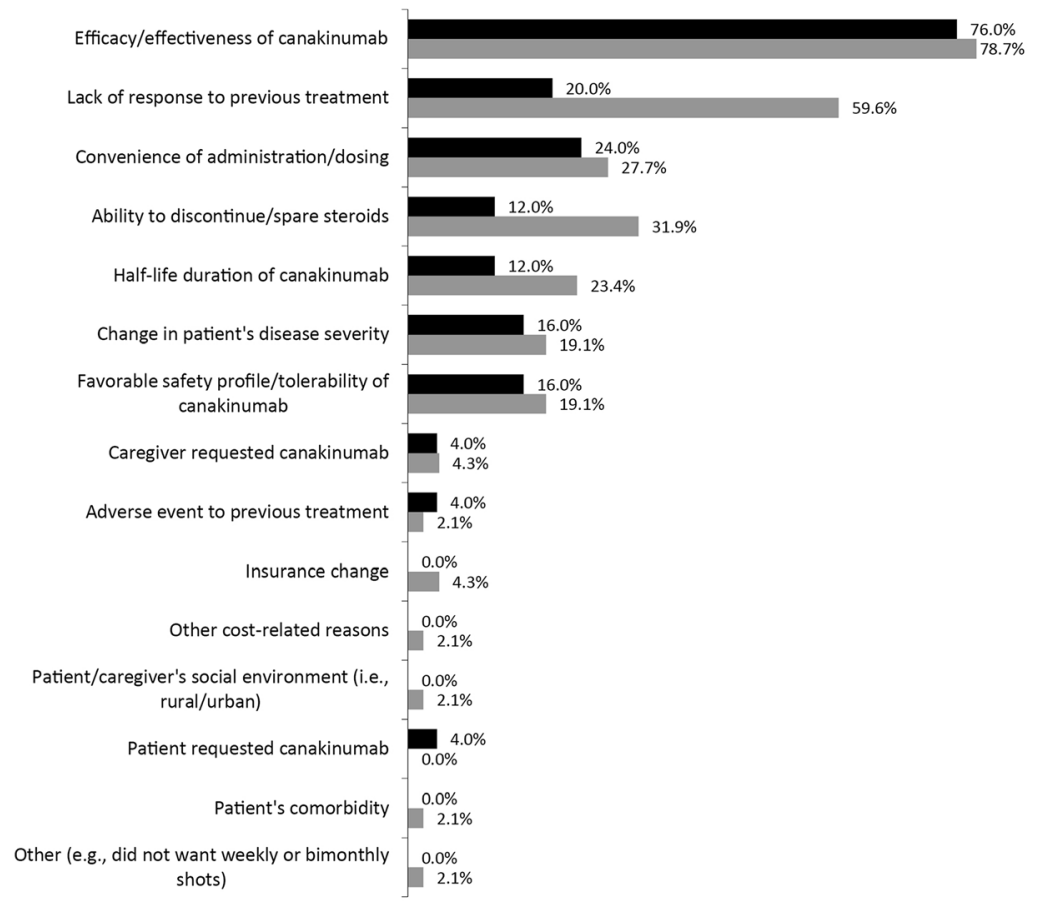


4Fig. 2 Reasons for canakinumab initiation among patients with SJIA/AOSD: a Children and adults, b By treating physician subspecialty. Note: More than one reason per patient possible. AOSD Adult-onset Still's disease, $N$ total number of patients in the respective category, SJIA systemic juvenile idiopathic arthritis

canakinumab (23.4 vs. $12.0 \%$ ) were the more frequent reasons for canakinumab initiation versus those treated by pediatric subspecialists (Fig. 2b).

\section{Sensitivity Analysis}

The results of the sensitivity analysis performed by excluding the patients with AOSD and those whose age at symptom onset was unavailable $(n=54)$ were similar to results of the overall population $(n=72)$, with no effect on study conclusions (data not shown and available upon request).

The results of the second sensitivity analysis performed only on those patients with AOSD $(n=13)$ suggested that etanercept $(50.0 \%)$ and anakinra $(33.3 \%)$ were the commonest treatment prior to canakinumab, and the availability of a new treatment $(38.5 \%)$ and half-life duration of the agent $(23.1 \%)$ were the most common reasons for treatment discontinuation. The initial dosing frequency of canakinumab was $\mathrm{q} 4 \mathrm{w}$ in majority of patients $(76.9 \%)$, with an average dose of $126.0 \mathrm{mg}$ (or $4.0 \mathrm{mg} / \mathrm{kg}$ ). Physician perceived/experienced efficacy/effectiveness and lack-of-response to previous treatment (each 61.5\%) were the most cited reasons for canakinumab initiation in patients with AOSD, followed by ability to discontinue/spare steroids (30.8\%; ESM Tables S3-S6).

\section{DISCUSSION}

This retrospective review of 72 medical charts of patients with SJIA and AOSD revealed that patients commonly received etanercept, anakinra, adalimumab and tocilizumab as the treatment directly preceding canakinumab initiation and that these were discontinued mainly due to lack of efficacy/effectiveness and availability of a new treatment. Physician perceived/experienced efficacy/effectiveness of canakinumab, lack-of-response to previous treatment and convenient administration/dosing were the most common reasons for canakinumab initiation. To the authors' knowledge, this is the first study providing insights on how canakinumab is initiated in US clinical practice and physicians' treatment decision-making behavior for patients with Still's disease.

Based on the age at canakinumab initiation, a majority of patients in this study were children (61.1\%). This pattern was expected as the literature indicates that SJIA can manifest at any point until the age of 16 years, although symptoms usually occur in children aged $\leq 2$ years, and up to $30.0 \%$ still experience symptoms 10 years after their first symptoms [5]. The onset of AOSD by definition occurs after 16 years of age and its incidence is lower than that of SJIA [4].

The most common category of treating physician was rheumatologist, specifically adult subspecialists. Only $32.6 \%$ of treating physicians were pediatric subspecialists; therefore, limited availability of pediatric subspecialists with unequal geographic distribution, longer waiting time for a visit and significant travel distances could be, in part, the reasons for restricted access to pediatric subspecialty care [17]. Consequently, the children and their parents/caregivers may instead seek care from physicians with an adult subspecialty, which was also observed in this study, with $61.4 \%$ of children treated by adult subspecialists. Our findings were not directly comparable with those reported in the literature because evidence on treatment patterns by treating physician subspecialty is lacking.

We observed that $11.0-28.0 \%$ of patients were receiving tocilizumab, adalimumab, anakinra and etanercept directly preceding canakinumab initiation. A cross-sectional analysis of data from the Childhood Arthritis and Rheumatology Research Alliance (CARRA) Registry in the USA, including a subset of 246 patients with SJIA, reported that $46.0 \%$ of patients with SJIA received tumor necrosis factor alpha (TNF- $\alpha$ ) inhibitor and $39.0 \%$ received 
IL-1 inhibitor [18]. Another study from the USA which analyzed claims data of 106 patients with AOSD reported the use of anakinra in $25.0 \%$ of patients, and etanercept and adalimumab in 9.0-10.0\% [19]. Our study also showed similar results. Nonetheless, the use of etanercept, anakinra and adalimumab in patients with SJIA were "off-label." Thus, more awareness is needed about the use of therapies which have specifically been approved by the US Food and Drug Administration for SJIA. IL-1 and IL-6 inhibitors are also presumed to be more beneficial than TNF- $\alpha$ inhibitors in SJIA because of their major roles in SJIA pathogenesis. Of note, IL-1 inhibition appears to be exceptionally effective strategy in new-onset SJIA, and there appears to be a "window of opportunity" to introduce these therapies early to alter the disease course to avoid the development of chronic arthritis [20]. Evidence suggests that IL-1 inhibitors when given as first-line treatment to patients with SJIA provided excellent outcomes, better than those observed when initiated later in the disease course and helped prevent patients from receiving corticosteroids [21-23].

Overall, the lack of efficacy/effectiveness and availability of a new treatment were the most prominent reasons for treatment discontinuation prior to canakinumab. Per specific biologic, the lack of efficacy/effectiveness was the reason for discontinuation of adalimumab, tocilizumab and etanercept among all or a majority of the patients. Short half-life and high frequency of injection were reasons for anakinra discontinuation, while AEs were the reason for etanercept discontinuation. Our observations were consistent with findings from the literature. A Latin American study on clinical remission in patients with SJIA reported that $49.0 \%$ patients on etanercept were switched to another antiTNF agent due to no response or lack of sufficient response [24]. A study from the UK characterizing the prescription patterns of a second biologic in a cohort of patients with SJIA reported that patients receiving anakinra, etanercept, tocilizumab and adalimumab were switched to another biologic mainly due to inefficacy and AEs, with adherence to treatment also being a reason for switching among patients on anakinra [25].
In the USA, canakinumab is approved for Still's disease, including SJIA and AOSD, at the dosing regimen of $4.0 \mathrm{mg} / \mathrm{kg}$ (with maximum of $300.0 \mathrm{mg}$ ) for patients with a body weight of $\geq 7.5 \mathrm{~kg}$ [11]. We observed that the average initial dose of canakinumab was different in children and adults (3.3 vs. $2.3 \mathrm{mg} / \mathrm{kg})$, with two dosing frequencies ( $\mathrm{q} 4 \mathrm{w}$ and $\mathrm{q} 8 \mathrm{w}$ ) commonly employed despite only $\mathrm{q} 4 \mathrm{w}$ dosing being approved [11]. While the canakinumab $\mathrm{q} 4 \mathrm{w}$ dose was more common among adults, the $\mathrm{q} 8 \mathrm{w}$ dose was more common among children. As our study was not designed to follow-up the patient after canakinumab initiation, the exact reasons for change in the initial/maintenance dosing from the approved label could not be ascertained and further research is suggested.

Our study revealed that physician perceived/experienced efficacy/effectiveness and lack-of-response to previous treatment were the primary reasons for canakinumab initiation among patients with Still's disease. These findings were not directly comparable to those reported in the literature due to a lack of evidence from the USA; however, they are in agreement with those from a study carried out in the UK [25]. This UK study (as described above) reported that patients with SJIA receiving a biologic as the first-line agent were switched to canakinumab mainly due to inefficacy of previous treatment [25]. We observed differences in the reasons for canakinumab initiation among children and adults, as well as by subspecialty of the treating physician. Further research is needed to better understand the reasons behind this prescription behavior.

This study has certain limitations. There is the potential for inaccurate data recorded in the primary charts. There is the possibility of errors introduced during data entry by physicians although logic checks were implemented to minimize these errors. The study may have been affected by reporting bias (e.g. bias in favor of specific practice per guideline recommendations) or recall bias and non-random missing data (e.g. specifically ignoring a particular answer option across questions). The large panel of physicians utilized to invite participants comprises one of the most comprehensive physician panels in the USA for research 
purposes. However, the physicians' response rates were low, which could have introduced selection bias as the physicians who responded to the invitation to participate in the study may be different than those who did not respond. Most physicians were in private practice, which also could have introduced a bias to the type of patient included in this study. Lastly, the study was not intended to collect the information on why patients did not prescribe or use canakinumab. Nonetheless, in a panel-based study, approaching physicians by e-mail is common, and this approach has resulted in a large sample given that SJIA/AOSD is a rare disease. Furthermore, the study provided meaningful and timely clinical data on patients with SJIA/AOSD who were prescribed canakinumab. The results from this study might not be generalizable to a greater population due to the specific patient and physician inclusion criteria.

\section{CONCLUSIONS}

The study results revealed the common factors leading to canakinumab initiation among patients with SJIA/AOSD in US clinical practice. Physician perceived/experienced efficacy/effectiveness of canakinumab and lack-of-response to previous treatment were the primary reasons for canakinumab initiation among this patient population. The most common reasons for canakinumab initiation among children versus adults were physician perceived/experienced efficacy/effectiveness and convenient administration/dosing of canakinumab, whereas lackof-response to previous treatment and ability to discontinue/spare steroids were the most frequent reasons among adults. In patients treated by adult subspecialists, lack-of-response to previous treatment, ability to discontinue/spare steroids and half-life of canakinumab were the main reasons for canakinumab initiation versus those treated by pediatric subspecialists. Further research is suggested to understand the factors behind these differences by patient age and physician subspecialty.

\section{ACKNOWLEDGEMENTS}

Funding. Sponsorship for this study and Rapid Service Fee were funded by Novartis Pharmaceuticals Corporation, East Hanover, NJ, USA.

Editorial Assistance. Editorial assistance in the preparation of this article was provided by Amit Pagada of Novartis Healthcare Pvt. Ltd., India.

Authorship. All named authors meet the International Committee of Medical Journal Editors (ICMJE) criteria for authorship for this article, take responsibility for the integrity of the work as a whole, and have given their approval for this version to be published.

Authors' Contributions. Peter Hur, Raluca Ionescu-Ittu, Ameur M. Manceur, Kathleen G. Lomax, Jordan Cammarota, Jipan Xie, Navneet Sanghera and Alexei A. Grom contributed to conception and design of the work. Raluca Ionescu-Ittu, Ameur M. Manceur, Jordan Cammarota, Jipan Xie and Nina Kim conducted data collection and performed data analysis. Peter Hur, Esther Yi, Kathleen G. Lomax, Raju Gautam, Navneet Sanghera and Alexei A. Grom contributed to data interpretation. Peter Hur, Esther Yi, Kathleen G. Lomax, Raju Gautam, Priscila Nakasato, Navneet Sanghera and Alexei A. Grom assisted in drafting the manuscript. All authors critically revised the manuscript and read and approved the final manuscript for submission.

Prior Presentation. This research work was presented at the 2019 American College of Rheumatology/The Association of Rheumatology Professionals (ACR/ARP) Annual Meeting, held in November 2019 at Atlanta, GA, USA.

Disclosures. Peter Hur was an employee of Novartis Pharmaceuticals Corporation, USA at the time of conduct of this study and is currently an employee of Pfizer Inc., USA. Esther Yi, Priscila Nakasato and Navneet Sanghera are employees of Novartis Pharmaceuticals 
Corporation, USA. Ameur M. Manceur, Jordan Cammarota and Jipan Xie are employees of Analysis Group, Inc., a consulting company that has provided paid consulting services to Novartis for the execution of this study. Raluca Ionescu-Ittu was an employee of Analysis Group, Inc. at the time of conduct of this study and is currently an employee of Statlog Inc., Canada. Kathleen G. Lomax was an employee of Novartis Pharmaceuticals Corporation, USA at the time of conduct of this study and is currently an employee of Janssen Pharmaceuticals, USA. Raju Gautam was an employee of Novartis Healthcare Pvt. Ltd., India at the time of conduct of this study and is currently an employee of Eversana, India. Nina Kim was associated with the Baylor Scott and White Medical Center, Texas and University of Texas, USA and provided services to Novartis as a postdoctoral fellow. Alexei A. Grom is an employee of Children's Hospital Medical Center, USA and has received speaker/consulting fees from Novartis and Roche, and has been involved in research collaboration for Sobi (NovImmune).

Compliance with Ethics Guidelines. Ethics approval was obtained from the New England Independent Review Board (NEIRB, \#120180302) prior to the initiation of data collection. The study was conducted in accordance with the Helsinki Declaration of 1964 and its later amendments.

Data Availability. The dataset generated during and/or analyzed during the current study are available from the corresponding author on reasonable request.

Open Access. This article is licensed under a Creative Commons Attribution-NonCommercial 4.0 International License, which permits any non-commercial use, sharing, adaptation, distribution and reproduction in any medium or format, as long as you give appropriate credit to the original author(s) and the source, provide a link to the Creative Commons licence, and indicate if changes were made. The images or other third party material in this article are included in the article's Creative Commons licence, unless indicated otherwise in a credit line to the material. If material is not included in the article's Creative Commons licence and your intended use is not permitted by statutory regulation or exceeds the permitted use, you will need to obtain permission directly from the copyright holder. To view a copy of this licence, visit http://creativecommons.org/licenses/by$\mathrm{nc} / 4.0 /$.

\section{REFERENCES}

1. Shenoi S, Wallace CA. Diagnosis and treatment of systemic juvenile idiopathic arthritis. J Pediatr. 2016;177:19-26.

2. Grevich S, Shenoi S. Update on the management of systemic juvenile idiopathic arthritis and role of IL1 and IL-6 inhibition. Adolesc Health Med Ther. 2017;8:125-35.

3. Shenoi S, Horneff G, Cidon M, et al. The burden of systemic juvenile idiopathic arthritis for patients and caregivers: an international survey and retrospective chart review. Clin Exp Rheumatol. 2018;36(5):920-8.

4. Magadur-Joly G, Billaud E, Barrier JH, et al. Epidemiology of adult Still's disease: estimate of the incidence by a retrospective study in west France. Ann Rheum Dis. 1995;54(7):587-90.

5. Gurion R, Lehman TJ, Moorthy LN. Systemic arthritis in children: a review of clinical presentation and treatment. Int J Inflam. 2012;2012: 271569.

6. Mellins ED, Macaubas C, Grom AA. Pathogenesis of systemic juvenile idiopathic arthritis: some answers, more questions. Nat Rev Rheumatol. 2011;7(7):416-26.

7. Moorthy LN, Peterson MG, Hassett AL, Lehman TJ. Burden of childhood-onset arthritis. Pediatr Rheumatol Online J. 2010;8:20.

8. Beukelman T, Patkar NM, Saag KG, et al. 2011 American College of Rheumatology recommendations for the treatment of juvenile idiopathic arthritis: initiation and safety monitoring of therapeutic agents for the treatment of arthritis and systemic features. Arthritis Care Res. 2011;63(4): 465-82.

9. US Food and Drug Administration. Actemra (tocilizumab). Prescribing Information. 2021. https:// www.accessdata.fda.gov/drugsatfda_docs/label/ 2021/125472s044lbl.pdf. Accessed 15 Sept 2021. 
10. European Medicines Agency. Ilaris (canakinumab) European public assessment reports summary. 2020. Available from: https://www.ema.europa.eu/ en/medicines/human/EPAR/ilaris. Accessed $15 \mathrm{Sept}$ 2021.

11. US Food and Drug Administration. Ilaris (Canakinumab). Prescribing Information. 2020. https:// www.accessdata.fda.gov/drugsatfda_docs/label/ 2020/125319s100lbl.pdf. Accessed 15 Sept 2021.

12. Ruperto N, Brunner HI, Quartier P, et al. Two randomized trials of canakinumab in systemic juvenile idiopathic arthritis. NEJM. 2012;367(25):2396-406.

13. Ruperto N, Quartier P, Wulffraat N, et al. A phase II, multicenter, open-label study evaluating dosing and preliminary safety and efficacy of canakinumab in systemic juvenile idiopathic arthritis with active systemic features. Arthritis Rheum. 2012;64(2): 557-67.

14. Kedor C, Listing J, Zernicke J, et al. Canakinumab for treatment of adult-onset still's disease to achieve reduction of arthritic manifestation (CONSIDER): phase II, randomised, double-blind, placebo-controlled, multicentre, investigator-initiated trial. Ann Rheum Dis. 2020;79(8):1090-7.

15. Hur P, Lomax KG, Ionescu-Ittu R, et al. Reasons for canakinumab initiation among patients with periodic fever syndromes: a retrospective medical chart review from the United States. Pediatr Rheumatol Online J. 2021;19(1):143.

16. Efthimiou P, Paik PK, Bielory L. Diagnosis and management of adult onset Still's disease. Ann Rheum Dis. 2006;65(5):564-72.

17. Ray KN, Kahn JM, Miller E, Mehrotra A. Use of adult-trained medical subspecialists by children seeking medical subspecialty care. J Pediatr. 2016;176:173-81.e1.
18. Beukelman T, Ringold S, Davis TE, et al. Diseasemodifying antirheumatic drug use in the treatment of juvenile idiopathic arthritis: a cross-sectional analysis of the CARRA Registry. J Rheumatol. 2012;39(9):1867-74.

19. Lenert A, Oh G, Ombrello MJ, Kim S. Clinical characteristics and comorbidities in adult-onset Still's disease using a large US administrative claims database. Rheumatology (Oxford). 2020;59: 1725-33.

20. Nigrovic PA. Review: is there a window of opportunity for treatment of systemic juvenile idiopathic arthritis? Arthritis Rheumatol. 2014;66(6):1405-13.

21. Horneff G, Peitz J, Kekow J, Foell D. Canakinumab for first line steroid-free treatment in a child with systemic-onset juvenile idiopathic arthritis. Scand J Rheumatol. 2017;46(6):500-1.

22. Nigrovic PA, Mannion M, Prince FH, et al. Anakinra as first-line disease-modifying therapy in systemic juvenile idiopathic arthritis: report of forty-six patients from an international multicenter series. Arthritis Rheum. 2011;63(2):545-55.

23. Vastert SJ, de Jager W, Noordman BJ, et al. Effectiveness of first-line treatment with recombinant interleukin-1 receptor antagonist in steroid-naive patients with new-onset systemic juvenile idiopathic arthritis: results of a prospective cohort study. Arthritis Rheumatol. 2014;66(4):1034-43.

24. Russo RA, Katsicas MM. Clinical remission in patients with systemic juvenile idiopathic arthritis treated with anti-tumor necrosis factor agents. J Rheumatol. 2009;36(5):1078-82.

25. Kearsley-Fleet L, Davies R, Baildam E, et al. Factors associated with choice of biologic among children with Juvenile Idiopathic Arthritis: results from two UK paediatric biologic registers. Rheumatology (Oxford). 2016;55(9):1556-65. 